\title{
Blood Cultures and Broad-Spectrum Antimicrobials are Essential for Patients with Out-of-Hospital Cardiac Arrest
}

Ching-Chi Lee ( $\nabla$ chichingbm85@gmail.com )

National Cheng Kung University Hospital https://orcid.org/0000-0002-4949-2742

\section{Po-Lin Chen}

National Cheng Kung University Hospital

Ming-Yuan Hong

National Cheng Kung University Hospital

Chih-Hao Lin

National Cheng Kung University Hospital

Wen-Chien Ko

National Cheng Kung University Hospital

\section{Research}

Keywords: bacteremia, out-of-hospital cardiac arrest, empirical antibiotic

Posted Date: September 28th, 2020

DOI: https://doi.org/10.21203/rs.3.rs-82686/v1

License: (c) (1) This work is licensed under a Creative Commons Attribution 4.0 International License. Read Full License 


\section{Abstract}

Background: Etiologies of out-of-hospital cardiac arrest (OHCA) have been majorly focused on cardiac origins. Little is known regarding the role of bloodstream infections (BSIs) in OHCA episodes. Our aim was to disclose clinical features and incidences of BSIs and the survival benefit of prompt administration of appropriate antimicrobial therapy (AAT) in adults with OHCA.

Methods: In the 10-year multicenter cohort, clinical information was retrospectively collected and causative microorganisms were prospectively stored for species identification and susceptibilities. The effect of delayed AAT administration on 30-day mortality was examined after adjustment for independent predictors of mortality, recognized by a multivariate regression analysis.

Results: Of 1,021,177 emergency department encounters, OHCA visits had a higher BSI incidence than non-OHCA visits $(413 / 3,429,12.0 \%$ vs. $7,429 / 242,302,3.1 \%$; $P<0.001)$. Compared with the matched nonOHCA $(2,478)$ patients, OHCA $(413)$ patients experienced more bacteremic episodes due to lower respiratory tract infections, fewer urosepsis events, fewer Escherichia coli bacteremia, and more streptococcal and anaerobes bacteremia. More antimicrobial-resistant organisms, such as methicillinresistant Staphylococcus aureus and extended-spectrum beta-lactamase-producing Enterobacteriaceae, were evident in OHCA patients. Notably, each hour delay in AAT administration was associated with an average increase of $0.8 \%$ in crude 30 -day mortality rates (adjusted odds ratio [AOR], $1.008 ; P=0.04$ ) in OHCA patients, $7 \%(A O R, 1.007 ; P<0.001)$ in critically ill patients without OHCA, and $3 \%(A O R, 1.003 ; P<$ $0.001)$ in less critically ill patients.

Conclusions: BSIs should be considered in patients experiencing OHCA; bacteremia sources, causative microorganisms, and antimicrobial susceptibilities differed between the OHCA and non-OHCA patients. The incorporation of blood culture samplings and rapid initiation of broad-spectrum antimicrobial therapy as the first-aids is essential for OHCA patients.

\section{Introduction}

Bloodstream infections (BSIs) are associated with substantial morbidity and mortality that cause a significant burden of healthcare costs [1]. Community-onset bacteremia was reported to have an annual incidence of $0.14 \%-0.82 \%[2,3]$ and the short-term fatality rate of up to $41.5 \%$ [4]. In addition to hemodynamic support, prompt administration of appropriate antimicrobials has been evidenced to provide survival benefits for patients with BSIs, particularly those who are critically ill [4-7].

Despite globe variation in the incidence and survival rate, out-of-hospital cardiac arrest (OHCA) heralded a universally miserable outcome and its main etiology was originally considered to be of cardiac origins [8]. Although many studies have reported non-cardiac causes of OHCA [9-11], little is known regarding the prevalence and characteristic of BSIs in patients with OHCA[12]. Therefore, emergency department (ED) physicians do not include blood cultures to be a routine test for patients with OHCA and do not administer antimicrobial therapy as the first aids to such patients. Accordingly, we aimed to elucidate the etiological 
role of BSIs and the potential benefit of early administration of appropriate antimicrobials in adults experiencing OHCA.

\section{Materials And Methods Study design and sites}

The multicenter retrospective study was conducted from January 2012 to December 2017 at the EDs of three hospitals with 1,200, 460, and 380 beds, respectively, in southern Taiwan. The targeted population was OHCA adults with community-onset bacteremia at the ED. All resuscitations were directed by boardcertified ED physicians and nurses following the contemporary guidelines of advanced cardiac life support (ACLS). For patient experiencing OHCA, their information prior to ED arrival was captured from an Utstein-style population database, a prospective registry database, in southern Taiwan, and partial information in this database has been published [13]. The study was approved by the institutional review boards of three study hospital and the requirement of informed consent was waived.

\section{Patient Population}

First, medical records of all ED visits during the study period were retrospectively reviewed in a computer database for the OHCA presentation. Patients with OHCA who had blood cultures sampled during or prior to resuscitation and those without OHCA patients who had blood cultures sampled during the ED stay were included. The medical records of adults with documented bacterial growth in blood cultures were carefully reviewed. Patients with contaminated blood cultures, those with hospital-onset bacteremia, and those transferred from other hospitals were excluded from this study. Second, to investigate the initial manifestation of bacteremia in patients with and without OHCA, adults with OHCA with records of community-onset bacteremia were included as the OHCA group. Six patients without OHCA who had community-onset bacteremia and temporally near ED arrival of the enrolled OHCA visit were matched as the non-OHCA comparators. Finally, to study the impact of delayed administration of appropriate antimicrobials on short-term prognoses, regardless of whether the patient experienced OHCA, patients with uncertain fatality or incomplete clinical information within 30 days of bacteremia onset were excluded.

\section{Data Collection}

For OHCA and non-OHCA patients, clinical information after ED arrival was collected by reviewing medical records by one board-certified ED physician and another infectious-disease clinician, and the discrepancy was solved by the discussion between the authors. Using a predetermined form, the capturing data included demographic information, vital signs, comorbidities, comorbidity severity (McCabe-Johnson classification), bacteremia severity (a Pitt bacteremia score) at onset, bacteremia sources, durations and types of antimicrobial therapy, and causative microorganisms. Moreover, frequencies of ED visits within six months before the onset of bacteremia, subsequent discharge through the ED or hospitalization, and 
short-term outcomes were obtained. The study primary outcome was crude 30-day mortality after bacteremia onset (i.e., ED arrival).

Focusing on OHCA patients, prehospital data included the witness status, the presence of bystander cardiopulmonary resuscitation (CPR) and witnessed cardiac arrest, the response time (defined as the period between the ambulance departure from the fire station and the arrival at the scene) of the emergency medical service (EMS), the EMS scene time (defined as the gap between the ambulance arrival at the scene and the ambulance departure from the scene), and the EMS transport time (defined as the period between the ambulance departure from the scene and ED arrival).

\section{Microbiological methods}

After sampling, blood cultures were incubated in a BACTEC 9240 instrument (Becton Dickinson Diagnostic Systems, Sparks, MD, USA) for 5 days at $35^{\circ} \mathrm{C}$. The causative microorganisms were identified by a semiautomated system (Vitek 2 system, bioMe'rieux, Durham, NC), and were stored for further susceptibility testing using disk diffusion method for aerobes or agar dilution method for anaerobes, if the susceptibility results of administrated antimicrobials were provided by the study hospital.

To further investigate the incidence of antimicrobial-resistant organisms (AROs), antimicrobial susceptibility of ampicillin was tested for enterococci, cefoxitin for Staphylococcus aureus (to reflect methicillin susceptibility), penicillin for streptococci, and ampicillin/sulbactam for anaerobes. For the isolates of Escherichia coli, Klebsiella species, and Proteus mirabilis (EKP), susceptibilities to cefepime and levofloxacin were tested and the production of extended-spectrum beta-lactamases (ESBLs) was determined by the phenotypic confirmatory test of cephalosporin-clavulanate combination disks [14]. All susceptibilities were interpreted by the contemporary CLSI recommendation [15].

\section{Definitions}

Cardiac arrest was diagnosed based on the updated definition issued by the American Heart Association and the International Consensus Conference on Cardiopulmonary Resuscitation [16]. The term 'bacteremia' is generally understood to mean the bacterial growth of blood cultures obtained from central or peripheral venipuncture, after the exclusion of contaminant sampling. In accordance with previous criteria [17], blood cultures with the growth of coagulase-negative staphylococci, Bacillus spp., Micrococcus spp., Propionibacterium spp., or Gram-positive bacilli, were considered as contaminant samplings. Community-onset bacteremia indicates that the place of the bacteremia onset is the community, and includes health care-associated and community-acquired bacteremia $[5,7]$.

Polymicrobial bacteremia was defined as the isolation of more than one microbial species from a single bacteremic episode, whereas the other was regarded as monomicrobial bacteremia.

As previous descriptions [5, 7], antimicrobial therapy was regarded as appropriate, if the following two criteria were fulfilled: (i) the route and dosage of antimicrobials was administered as recommended in the 2020 Sanford Guide [18]; (ii) antimicrobials administered were in vitro active against all causative microorganisms of bacteremia, based on the contemporary CLSI breakpoints [15]. The time-to- 
appropriate antibiotic (TAA) was defined as the time gap between the first dose of appropriate antibiotic administration and bacteremia onset $[5,7]$.

Comorbidities were defined as described previously [19], and the comorbidity severity was graded as the classification system proposed by McCabe and Johnson [20]. BSI severity was graded in accordance with a Pitt bacteremia score using a previously validated scoring system during the first 24 hours after ED arrival $[5,7]$. Patients having a high Pitt bacteremia score $(\geq 4)$ was categorized as the critical illness, whereas those with a Pitt bacteremia score of $<4$ as the less critical illness. Bacteremia sources were clinically identified according to one of the following criteria: the presence of an active infection site coincident with bloodstream infections; and the isolation of a microorganism from other clinical specimens before or on the same date as that of bacteremia onset [21]. For complicated bacteremia, the removal of infected hardware, drainage of infected fluid collections, or resolution of obstruction for biliary or urinary sources was referred as appropriate control of bacteremia source [22]. Crude mortality was equated with the death from all causes [7].

\section{Statistical analyses}

The Statistical Package for the Social Science for Windows (Version 23.0; Chicago, IL, USA) was used for statistical analyses. Categorical variables were compared using the Fisher exact or Pearson chi-square test. Continuous variables were presented as medians (interquartile ranges, IQRs) and were compared using an independent $t$ test or Mann-Whitney $\mathrm{U}$ test.

To investigate the independent effect of the TAA (each hour) on 30-day mortality, the variables of 30-day crude mortality, recognized through the univariate analysis with a $P$ value of $\leq 0.05$, or 0.1 for a small patient population, and the TAA were together included in a stepwise and backward multivariable logistic regression model. A two-sided $P$ value of $\leq 0.05$ was considered statistically significant.

\section{Results}

\section{Patient demographics}

During the ten-year study period, OHCA patients accounted for $0.60 \%(6,142)$ of the total $1,021,177 \mathrm{ED}$ visits. Blood cultures were sampled in $55.8 \%(3,429)$ of OHCA patients, and in contrast only $23.9 \%$ $(242,302)$ of non-OHCA patients $(P<0.001)$. The contamination rate of blood cultures $(9.8 \%, 335 / 3,429$ vs. $1.7 \%, 4,221 / 242,302 ; P<0.001)$ and true bacteremia incidence $(12.0 \%, 413 / 3,429$ vs. $3.1 \%$, $7,429 / 242,302 ; P<0.001)$ in OHCA patients was significantly higher than that in non-OHCA patients. Based on the inclusion and exclusion criteria, the study cohort of community-onset bacteremia consisted of 413 OHCA patients and 2,478 matched non-OHCA patients at the case-matched control ratio of 1:6 (Fig. 1).

Of 413 OHCA patients with BSIs, their median (IQR) age was 76 (60-83) years, and 219 (53.0\%) were male. The median (IQR) length of ED stay was 5.0 (2.0-17.8) hours. The patients with bystander CPR and 
witnessed cardiac arrest respectively accounted for $53.0 \%$ (219 patients) and $46.5 \%$ (192); the median (IQR) of the response time, the scene time, and the transport time offered by the EMS was 5.6 (4.5-7.3), 10.1 (8.0-13.0), and 5.0 (3.0-7.8) minutes, respectively. The majority $(357,86.4 \%)$ of OHCA patients had sustained ( $\geq 2$ hours) spontaneous circulation. After resuscitation, $227(55.0 \%)$ patients were admitted to intensive care units (ICUs), 176 (42.6\%) died at the ED, and ten (2.4\%) were admitted to general wards.

Among 2,478 matched non-OHCA patients, the elderly accounted for $60.9 \%$ (1,509 patients), and $51.2 \%$ (1269) were male. The median (IQR) length of ED stay was 15.4 (5.6-26.0) hours; most patients $(2,058$, 83.1\%) were admitted to general wards, 227 (9.2\%) to ICUs, 50 (2.0\%) died at the ED, and 131 (5.3\%) were directly discharged from the ED and followed up in the out-patient clinic. However, 28 non-OHCA patients with uncertain outcome within 30 days after bacteremia onset were noticed.

\section{Clinical characteristics and outcomes of patients with and without OHCA}

The comparisons of clinical variables between patients with and without OHCA, in terms of demographics, bacteremia severity, types and severity of comorbidities, timing of appropriate antimicrobial administration, and patient outcomes, were examined by the univariate analyses (Table 1). OHCA patients were more likely to be the elderly, nursing home residents, bed-ridden status, and to have a critical illness (Pitt bacteremia score $\geq 4$ ), polymicrobial bacteremia, bacteremia due to lower respiratory tract infections, high leucocytes or c-reactive protein, comorbidities of diabetes mellitus, chronic obstructive pulmonary diseases, neurological, psychological, urological, or coronary artery diseases. In addition, OHCA patients had more prior ED visits. However, bacteremia due to urinary tract, intraabdominal, biliary tracts, or skin and soft-tissue infections, and comorbid liver cirrhosis were less common in OHCA patients. The longer TAA and more frequencies of ED visits within prior six months was observed in OHCA patients. Not surprisingly, the crude 7-day, 15-day, or 30-day mortality rate in OHCA patients was higher than that in non-OHCA patients. 
Table 1

Clinical manifestations and outcomes of community-onset bacteremia in adults with and without out-ofhospital cardiac arrest (OHCA)*.

\begin{tabular}{|c|c|c|c|}
\hline \multirow[t]{3}{*}{ Variables } & \multicolumn{2}{|c|}{ Patient numbers (\%) } & \multirow{3}{*}{$\begin{array}{l}P \\
\text { value }\end{array}$} \\
\hline & OHCA & Non-OHCA & \\
\hline & $n=413$ & $n=2,450$ & \\
\hline \multicolumn{4}{|l|}{ Patient demographics } \\
\hline Gender, male & $219(53.0)$ & $1,253(51.1)$ & 0.48 \\
\hline The elderly, $\geq 65$ years & $290(70.2)$ & $1496(61.1)$ & $\stackrel{<}{0.001}$ \\
\hline Nursing-home residents & $64(15.5)$ & $131(5.3)$ & $\stackrel{<}{0.001}$ \\
\hline Bed-ridden status & $163(39.5)$ & $301(12.3)$ & $\hat{0} .001$ \\
\hline ED visits within prior 6 months, median (IQR) & $1(1-3)$ & $0(0-1)$ & $\dot{\delta} 001$ \\
\hline Polymicrobial bacteremia & $90(21.8)$ & $233(9.5)$ & $\stackrel{<}{0.001}$ \\
\hline \multicolumn{4}{|l|}{ Major sources of bacteremia } \\
\hline Lower respiratory tract infections & $260(63.0)$ & $319(13.0)$ & $\hat{0.001}$ \\
\hline Urinary tract infections & $27(6.5)$ & $823(33.6)$ & $\hat{0} .001$ \\
\hline Skin and soft-tissue infections & $20(4.8)$ & $246(10.0)$ & 0.001 \\
\hline Intra-abdominal infections & $13(3.1)$ & $310(12.7)$ & $\dot{\delta} 001$ \\
\hline Biliary tract infections & $7(1.7)$ & $223(9.1)$ & $\hat{0} .001$ \\
\hline Uncertain focus & $52(12.6)$ & $29(1.2)$ & $\begin{array}{l}<.001 \\
0.01\end{array}$ \\
\hline $\begin{array}{l}\text { Ultimately or rapidly fatal comorbidities (McCabe- } \\
\text { Johnson classification) }\end{array}$ & $111(26.9)$ & $581(23.7)$ & 0.17 \\
\hline
\end{tabular}

$\mathrm{ED}=$ emergency department; $\mathrm{IQR}=$ interquartile range.

*Boldface indicates statistical significance with a $P$ value of $\leq 0.01$. 


\begin{tabular}{|c|c|c|c|}
\hline \multirow[t]{3}{*}{ Variables } & \multicolumn{2}{|c|}{ Patient numbers (\%) } & \multirow{3}{*}{$\begin{array}{l}P \\
\text { value }\end{array}$} \\
\hline & OHCA & Non-OHCA & \\
\hline & $n=413$ & $n=2,450$ & \\
\hline Neurological diseases & $194(47.0)$ & $545(22.2)$ & $\begin{array}{l}<.001 \\
0.01\end{array}$ \\
\hline Hypertension & $190(46.0)$ & $1,175(48.0)$ & 0.70 \\
\hline Diabetes mellitus & $175(42.4)$ & $904(36.9)$ & 0.03 \\
\hline Malignancy & $123(29.8)$ & $688(28.1)$ & 0.48 \\
\hline Chronic kidney diseases & $85(20.6)$ & $441(18.0)$ & 0.21 \\
\hline Coronary artery diseases & $56(13.6)$ & $235(9.6)$ & 0.01 \\
\hline Urological diseases & $41(9.9)$ & $167(6.8)$ & 0.02 \\
\hline Chronic obstructive pulmonary diseases & $33(8.0)$ & $121(4.9)$ & 0.01 \\
\hline Psychological diseases & $25(6.1)$ & $34(1.4)$ & $\begin{array}{l}<.001 \\
0.00\end{array}$ \\
\hline Liver cirrhosis & $24(5.8)$ & $315(12.9)$ & $\begin{array}{l}<.001 \\
0.001\end{array}$ \\
\hline Time-to-appropriate antibiotic, hours, median (IQR) & $\begin{array}{l}2.0(1.0- \\
56.0)\end{array}$ & $2.0(1.0-8.0)$ & $\begin{array}{l}<.001 \\
0.001\end{array}$ \\
\hline Pitt bacteremia score $\geq 4$ at ED arrival & $413(100)$ & $427(17.4)$ & $\begin{array}{l}<.001 \\
0.00\end{array}$ \\
\hline \multicolumn{4}{|l|}{ Laboratory data in the ED, median (IQR) } \\
\hline Leukocyte $\left(1,000 / \mathrm{mm}^{3}\right)$ & $\begin{array}{l}14.0(7.6- \\
20.6)\end{array}$ & $\begin{array}{l}11.5(7.5- \\
16.3)\end{array}$ & $\begin{array}{l}<.001 \\
0.001\end{array}$ \\
\hline C-reactive protein (mg/L), $n=2,734$ & $\begin{array}{l}83.1(38.3- \\
211.8)^{(3)}\end{array}$ & $\begin{array}{l}69.5(28.5- \\
185.5)^{-}\end{array}$ & $\begin{array}{l}<.001 \\
0.001\end{array}$ \\
\hline \multicolumn{4}{|l|}{ Crude mortality rates } \\
\hline 7-day & $306(74.1)$ & $147(6.0)$ & $\begin{array}{l}<.001 \\
0.00\end{array}$ \\
\hline 15-day & 344 (83.3) & $221(9.0)$ & $\begin{array}{l}<.001 \\
0.001\end{array}$ \\
\hline 30-day & $376(91.0)$ & $309(12.6)$ & $\begin{array}{l}<.001 \\
0.00\end{array}$ \\
\hline \multicolumn{4}{|l|}{$\mathrm{ED}$ = emergency department; IQR = interquartile range. } \\
\hline *Boldface indicates statistical significance with a $P$ & $\leq 0.01$ & & \\
\hline
\end{tabular}




\section{Microorganisms and susceptibilities in OHCA and non- OHCA Patients}

Because of the presence of 90 and 233 polymicrobial episodes in the OHCA and non-OHCA patients, 526 and 2,715 causative microorganisms were collected, respectively (Fig. 2). The leading five bacterial genera/species in OHCA patients were Streptococcus species (104 isolates, 19.8\%), Escherichia coli (87, $16.5 \%)$, Klebsiella species (77, 14.6\%), Staphylococcus aureus $(63,12.0 \%)$, and anaerobes $(48,9.1 \%)$. Of the latter, three major genera were Fusobacterium (18 isolates, 37.5\%), Prevotella (10, 20.8\%), and Peptostreptococcus $(8,16.7 \%)$. Otherwise, the leading five bacterial genera/species in non-OHCA patients were the same as those in OHCA patients, but their listing order differed. Of 114 anaerobes in non-OHCA patients, the leading genus was Bacteroides (67 isolates, 58.8\%). Of noted, more AROs, including MRSA, ampicillin-resistant enterococci, penicillin-resistant streptococci, ampicillin/sulbatam-resistant anaerobes, and ESBL-producing or levofloxacin-resistant EKP, were noted in OHCA patients (Fig. 3A).

Because nursing home residents only accounted for $15.5 \%$ and $5.3 \%$, of OHCA and non-OHCA patients, respectively, comparisons of antimicrobial resistance in some pathogens causing community-onset bacteremia among home-living patients with and without OHCA were demonstrated in Fig. 3B. Similarly, among OHCA patients, methicillin resistance was present in $46.0 \%$ (23 isolates) of $42 \mathrm{~S}$. aureus isolates, penicillin resistance in $17.9 \%$ (17) of 95 streptococcal isolates, and ESBL production or levofloxacin resistance in $12.8 \%$ (21) or $18.3 \%$ (30), respectively, of 164 EKP isolates. These figures were significantly higher than those in corresponding pathogens among non-OHCA patients (Fig. 3B).

\section{Impacts of the time-to-appropriate antibiotic on mortality of patients with varied bacteremia severity}

After the exclusion of patients with uncertain outcome, a positive trend of the TAA in 30-day mortality rate among OHCA patients $(\gamma=0.966, P=0.002)$, non-OHCA patients with the critical illness $(\gamma=0.995, P<$ $0.001)$, and the less critically ill patients $(y=0.960, P=0.002)$ was evidenced in Fig. 4.

For 413 patients with OHCA, the ambulance response time (mean, 400.1 vs. 406.1 seconds, $P=0.93$ ), EMS scene time (mean, 711.9 vs. 879.6 seconds, $P=0.82$ ), and EMS transport time (mean, 414.4 vs. 463.2 seconds, $P=0.84$ ) in the survivors and fatal patients was similar. However, clinical predictors of crude 30-day mortality in the univariate analyses, in terms of the elderly, bystander CPR, witnessed cardiac arrest, fatal comorbidities (McCabe-Johnson classification), and comorbid psychological diseases, were identified (Table 2). In the multivariate regression analyses, the length (measured by hours) of the TAA remained as the crucial determinant (adjusted odds ratio [AOR], $1.008 ; P=0.048$ ) of 30 day mortality. 
Table 2

Impacts of the time-to-appropriate antibiotic on 30-day prognoses in patients with community-onset bacteremia, categorized in out-of-hospital cardiac arrest (OHCA), critically ill (Pitt bacteremia score $\geq 4$ ) non-OHCA, and less critically ill (Pitt bacteremia score < 4) patients*.

\begin{tabular}{|c|c|c|c|c|c|c|}
\hline \multirow[t]{2}{*}{ Clinical variables } & \multicolumn{2}{|c|}{$\begin{array}{l}\text { Patient number } \\
\text { (\%) }\end{array}$} & \multicolumn{2}{|c|}{$\begin{array}{l}\text { Univariate } \\
\text { analysis }\end{array}$} & \multicolumn{2}{|l|}{$\begin{array}{l}\text { Multivariate } \\
\text { analysis }\end{array}$} \\
\hline & Death & Survival & $\begin{array}{l}\mathrm{OR}(95 \% \\
\mathrm{Cl})\end{array}$ & $\begin{array}{l}P \\
\text { value }\end{array}$ & $\begin{array}{l}\mathrm{AOR}(95 \% \\
\mathrm{Cl})\end{array}$ & $\begin{array}{l}P \\
\text { value }\end{array}$ \\
\hline OHCA patients $(n=413)$ & $\begin{array}{l}n= \\
376\end{array}$ & $n=37$ & & & & \\
\hline $\begin{array}{l}\text { Time-to-appropriate antibiotic } \\
\text { (hour) }\end{array}$ & - & - & - & - & $\begin{array}{l}1.008 \\
(1.000- \\
1.016)\end{array}$ & 0.048 \\
\hline $\begin{array}{l}\text { Bystander cardiopulmonary } \\
\text { resuscitation }\end{array}$ & $\begin{array}{l}192 \\
(51.1)\end{array}$ & $\begin{array}{l}27 \\
(73.0)\end{array}$ & $\begin{array}{l}0.39 \\
(0.18- \\
0.82)\end{array}$ & 0.01 & $\begin{array}{l}0.37 \\
(0.17- \\
0.83)\end{array}$ & 0.02 \\
\hline Witnessed cardiac arrest & $\begin{array}{l}169 \\
(44.9)\end{array}$ & $\begin{array}{l}23 \\
(66.2)\end{array}$ & $\begin{array}{l}0.50 \\
(0.25- \\
0.99)\end{array}$ & 0.045 & $\begin{array}{l}0.47 \\
(0.22- \\
0.99)\end{array}$ & 0.046 \\
\hline The elderly & $\begin{array}{l}270 \\
(71.8)\end{array}$ & $\begin{array}{l}20 \\
(54.1)\end{array}$ & $\begin{array}{l}2.17 \\
(1.09- \\
4.29)\end{array}$ & 0.02 & $\begin{array}{l}2.66 \\
(1.26- \\
5.66)\end{array}$ & 0.01 \\
\hline $\begin{array}{l}\text { Ultimately or rapidly fatal } \\
\text { comorbidities (McCabe-Johnson } \\
\text { classification) }\end{array}$ & $\begin{array}{l}110 \\
(29.3)\end{array}$ & $1(2.7)$ & $\begin{array}{l}14.89 \\
(2.02- \\
109.94)\end{array}$ & 0.001 & $\begin{array}{l}16.95 \\
(2.24- \\
128.18 .45)\end{array}$ & 0.006 \\
\hline \multicolumn{7}{|l|}{ Comorbidities } \\
\hline Malignancy & $\begin{array}{l}117 \\
(31.1)\end{array}$ & $6(16.2)$ & $\begin{array}{l}2.33 \\
(0.95- \\
5.75)\end{array}$ & 0.06 & NS & NS \\
\hline Psychological diseases & $\begin{array}{l}19 \\
(5.1)\end{array}$ & $6(16.2)$ & $\begin{array}{l}0.28 \\
(0.10- \\
0.74)\end{array}$ & 0.02 & NS & NS \\
\hline $\begin{array}{l}\text { Critically ill non-OHCA patients }(\mathrm{n}= \\
427)\end{array}$ & $\begin{array}{l}n= \\
166\end{array}$ & $\mathrm{n}=261$ & & & & \\
\hline $\begin{array}{l}\text { Time-to-appropriate antibiotic } \\
\text { (hour) }\end{array}$ & - & - & - & - & $\begin{array}{l}1.007 \\
(1.004- \\
1.009)\end{array}$ & $<.001$ \\
\hline
\end{tabular}

$\mathrm{AOR}=$ adjusted odds ratio; $\mathrm{Cl}=$ confidence interval; $\mathrm{COPD}=$ Chronic obstructive pulmonary diseases; NS = not significant (after processing the backward multivariate regression); OR = odds ratio.

* The time-to-appropriate antibiotic, a continuous variable, was included in the multivariable logistic regression model; boldface indicates statistical significance with a $P$ value of $\leq 0.01$ under the multivariate regression model. 


\begin{tabular}{|c|c|c|c|c|c|c|}
\hline \multirow[t]{2}{*}{ Clinical variables } & \multicolumn{2}{|c|}{$\begin{array}{l}\text { Patient number } \\
\text { (\%) }\end{array}$} & \multicolumn{2}{|c|}{$\begin{array}{l}\text { Univariate } \\
\text { analysis }\end{array}$} & \multicolumn{2}{|c|}{$\begin{array}{l}\text { Multivariate } \\
\text { analysis }\end{array}$} \\
\hline & Death & Survival & $\begin{array}{l}\text { OR }(95 \% \\
\mathrm{Cl})\end{array}$ & $\begin{array}{l}P \\
\text { value }\end{array}$ & $\begin{array}{l}\text { AOR }(95 \% \\
\text { Cl) }\end{array}$ & $\begin{array}{l}P \\
\text { value }\end{array}$ \\
\hline Gender, male & $\begin{array}{l}109 \\
(65.7)\end{array}$ & $\begin{array}{l}143 \\
(54.8)\end{array}$ & $\begin{array}{l}1.58 \\
(1.06- \\
2.36)\end{array}$ & 0.03 & NS & NS \\
\hline Inadequate source control & $\begin{array}{l}10 \\
(6.0)\end{array}$ & $7(2.7)$ & $\begin{array}{l}2.33 \\
(0.87- \\
6.24)\end{array}$ & 0.09 & $\begin{array}{l}2.98 \\
(1.03- \\
8.63)\end{array}$ & 0.045 \\
\hline \multicolumn{7}{|l|}{ Bacteremia sources } \\
\hline Urinary tract infections & $\begin{array}{l}17 \\
(10.2)\end{array}$ & $\begin{array}{l}86 \\
(33.0)\end{array}$ & $\begin{array}{l}0.23 \\
(0.13- \\
0.41)\end{array}$ & $\hat{0} .001$ & $\begin{array}{l}0.39 \\
(0.21- \\
0.72)\end{array}$ & 0.003 \\
\hline Lower respiratory tract infections & $\begin{array}{l}65 \\
(39.2)\end{array}$ & $\begin{array}{l}63 \\
(24.1)\end{array}$ & $\begin{array}{l}2.02 \\
(1.33- \\
3.08)\end{array}$ & 0.001 & $\begin{array}{l}1.77 \\
(1.09- \\
2.89)\end{array}$ & 0.02 \\
\hline $\begin{array}{l}\text { Ultimately or rapidly fatal } \\
\text { comorbidities (McCabe-Johnson } \\
\text { classification) }\end{array}$ & $\begin{array}{l}78 \\
(47.0)\end{array}$ & $\begin{array}{l}65 \\
(24.9)\end{array}$ & $\begin{array}{l}2.67 \\
(1.77- \\
4.04)\end{array}$ & <. 001 & $\begin{array}{l}2.43 \\
(1.55- \\
3.82)\end{array}$ & $\begin{array}{l}<.001 \\
0.001\end{array}$ \\
\hline \multicolumn{7}{|l|}{ Comorbidities } \\
\hline Malignancy & $\begin{array}{l}76 \\
(45.8)\end{array}$ & $\begin{array}{l}70 \\
(26.8)\end{array}$ & $\begin{array}{l}2.30 \\
(1.53- \\
3.47)\end{array}$ & $\begin{array}{l}< \\
0.001\end{array}$ & NS & NS \\
\hline Neurological diseases & $\begin{array}{l}51 \\
(30.7)\end{array}$ & $\begin{array}{l}106 \\
(40.6)\end{array}$ & $\begin{array}{l}0.65 \\
(0.43- \\
0.98)\end{array}$ & 0.04 & NS & NS \\
\hline Liver cirrhosis & $\begin{array}{l}27 \\
(16.3)\end{array}$ & $23(8.8)$ & $\begin{array}{l}2.01 \\
(1.11- \\
3.64)\end{array}$ & 0.02 & NS & NS \\
\hline Urological diseases & $\begin{array}{l}8 \\
(4.8)\end{array}$ & $\begin{array}{l}33 \\
(12.6)\end{array}$ & $\begin{array}{l}0.35 \\
(0.16- \\
0.78)\end{array}$ & 0.007 & NS & NS \\
\hline COPD & $\begin{array}{l}8 \\
(4.8)\end{array}$ & $\begin{array}{l}27 \\
(10.3)\end{array}$ & $\begin{array}{l}0.44 \\
(0.19- \\
0.99)\end{array}$ & 0.04 & $\begin{array}{l}0.41 \\
(0.17- \\
1.03)\end{array}$ & 0.06 \\
\hline
\end{tabular}

$\mathrm{AOR}=$ adjusted odds ratio; $\mathrm{Cl}=$ confidence interval; $\mathrm{COPD}=$ Chronic obstructive pulmonary diseases; $\mathrm{NS}=$ not significant (after processing the backward multivariate regression); OR = odds ratio.

* The time-to-appropriate antibiotic, a continuous variable, was included in the multivariable logistic regression model; boldface indicates statistical significance with a $P$ value of $\leq 0.01$ under the multivariate regression model. 


\begin{tabular}{|c|c|c|c|c|c|c|}
\hline \multirow[t]{2}{*}{ Clinical variables } & \multicolumn{2}{|c|}{$\begin{array}{l}\text { Patient number } \\
\text { (\%) }\end{array}$} & \multicolumn{2}{|c|}{$\begin{array}{l}\text { Univariate } \\
\text { analysis }\end{array}$} & \multicolumn{2}{|c|}{$\begin{array}{l}\text { Multivariate } \\
\text { analysis }\end{array}$} \\
\hline & Death & Survival & $\begin{array}{l}\text { OR }(95 \% \\
\mathrm{Cl})\end{array}$ & $\begin{array}{l}P \\
\text { value }\end{array}$ & $\begin{array}{l}\text { AOR }(95 \% \\
\text { Cl) }\end{array}$ & $\begin{array}{l}P \\
\text { value }\end{array}$ \\
\hline Less critically ill patients $(n=2,023)$ & $\begin{array}{l}n= \\
143\end{array}$ & $\begin{array}{l}n= \\
1880\end{array}$ & & & & \\
\hline $\begin{array}{l}\text { Time-to-appropriate antibiotic } \\
\text { (hour) }\end{array}$ & - & - & - & - & $\begin{array}{l}1.003 \\
(1.001- \\
1.005)\end{array}$ & $\hat{0.001}$ \\
\hline Gender, male & $\begin{array}{l}85 \\
(59.4)\end{array}$ & $\begin{array}{l}916 \\
(48.7)\end{array}$ & $\begin{array}{l}1.54 \\
(1.09- \\
2.18)\end{array}$ & 0.01 & NS & NS \\
\hline Nursing-home residents & $\begin{array}{l}16 \\
(11.2)\end{array}$ & $59(3.1)$ & $\begin{array}{l}3.89 \\
(2.18- \\
6.95)\end{array}$ & <. 001 & $\begin{array}{l}4.79 \\
(2.47- \\
9.31)\end{array}$ & $\begin{array}{l}<.001 \\
0.001\end{array}$ \\
\hline Polymicrobial bacteremia & $\begin{array}{l}21 \\
(14.7)\end{array}$ & $\begin{array}{l}143 \\
(7.6)\end{array}$ & $\begin{array}{l}2.09 \\
(1.28- \\
3.43)\end{array}$ & 0.003 & $\begin{array}{l}1.89 \\
(1.10- \\
3.23)\end{array}$ & 0.02 \\
\hline \multicolumn{7}{|l|}{ Bacteremia sources } \\
\hline Urinary tract infections & $\begin{array}{l}24 \\
(16.8)\end{array}$ & $\begin{array}{l}696 \\
(37.0)\end{array}$ & $\begin{array}{l}0.34 \\
(0.22- \\
0.54)\end{array}$ & <. 001 & $\begin{array}{l}0.49 \\
(0.30- \\
0.80)\end{array}$ & 0.005 \\
\hline Lower respiratory tract infections & $\begin{array}{l}35 \\
(24.5)\end{array}$ & $\begin{array}{l}156 \\
(8.3)\end{array}$ & $\begin{array}{l}3.58 \\
(2.37- \\
5.42)\end{array}$ & $\hat{0.001}$ & $\begin{array}{l}2.22 \\
(1.37- \\
3.58)\end{array}$ & 0.001 \\
\hline Intraabdominal infections & $\begin{array}{l}28 \\
(19.6)\end{array}$ & $\begin{array}{l}239 \\
(12.7)\end{array}$ & $\begin{array}{l}1.67 \\
(1.08- \\
2.58)\end{array}$ & 0.02 & NS & NS \\
\hline $\begin{array}{l}\text { Ultimately or rapidly fatal } \\
\text { comorbidities (McCabe-Johnson } \\
\text { classification) }\end{array}$ & $\begin{array}{l}83 \\
(58.0)\end{array}$ & $\begin{array}{l}355 \\
(18.9)\end{array}$ & $\begin{array}{l}5.94 \\
(4.18- \\
8.45)\end{array}$ & <. 001 & $\begin{array}{l}3.33 \\
(2.18- \\
5.08)\end{array}$ & $\dot{0} 001$ \\
\hline \multicolumn{7}{|l|}{ Comorbidities } \\
\hline Liver cirrhosis & $\begin{array}{l}41 \\
(28.7)\end{array}$ & $\begin{array}{l}224 \\
(11.9)\end{array}$ & $\begin{array}{l}2.97 \\
(2.02- \\
4.38)\end{array}$ & $\hat{0.001}$ & $\begin{array}{l}2.25 \\
(1.46- \\
3.46)\end{array}$ & $\dot{0} 001$ \\
\hline
\end{tabular}

$\mathrm{AOR}=$ adjusted odds ratio; $\mathrm{Cl}$ = confidence interval; $\mathrm{COPD}=$ Chronic obstructive pulmonary diseases; $\mathrm{NS}=$ not significant (after processing the backward multivariate regression); OR = odds ratio.

* The time-to-appropriate antibiotic, a continuous variable, was included in the multivariable logistic regression model; boldface indicates statistical significance with a $P$ value of $\leq 0.01$ under the multivariate regression model. 


\begin{tabular}{|c|c|c|c|c|c|c|}
\hline \multirow[t]{2}{*}{ Clinical variables } & \multicolumn{2}{|c|}{$\begin{array}{l}\text { Patient number } \\
\text { (\%) }\end{array}$} & \multicolumn{2}{|c|}{$\begin{array}{l}\text { Univariate } \\
\text { analysis }\end{array}$} & \multicolumn{2}{|l|}{$\begin{array}{l}\text { Multivariate } \\
\text { analysis }\end{array}$} \\
\hline & Death & Survival & $\begin{array}{l}\text { OR }(95 \% \\
\mathrm{Cl})\end{array}$ & $\begin{array}{l}P \\
\text { value }\end{array}$ & $\begin{array}{l}\text { AOR }(95 \% \\
\mathrm{Cl})\end{array}$ & $\begin{array}{l}P \\
\text { value }\end{array}$ \\
\hline Malignancy & $\begin{array}{l}84 \\
(58.7)\end{array}$ & $\begin{array}{l}458 \\
(24.4)\end{array}$ & $\begin{array}{l}4.42 \\
(3.12- \\
6.27)\end{array}$ & $<.001$ & $\begin{array}{l}2.22 \\
(1.46- \\
3.38)\end{array}$ & $<.001$ \\
\hline COPD & $\begin{array}{l}13 \\
(9.1)\end{array}$ & $73(3.9)$ & $\begin{array}{l}2.48 \\
(1.34- \\
4.59)\end{array}$ & 0.003 & $\begin{array}{l}2.14 \\
(1.07- \\
4.30)\end{array}$ & 0.03 \\
\hline \multicolumn{7}{|c|}{$\begin{array}{l}\text { AOR = adjusted odds ratio; } \mathrm{Cl}=\text { confidence interval; } \mathrm{COPD}=\text { Chronic obstructive pulmonary diseases; } \\
\mathrm{NS}=\text { not significant (after processing the backward multivariate regression); OR = odds ratio. }\end{array}$} \\
\hline \multicolumn{7}{|c|}{$\begin{array}{l}\text { * The time-to-appropriate antibiotic, a continuous variable, was included in the multivariable logistic } \\
\text { regression model; boldface indicates statistical significance with a } P \text { value of } \leq 0.01 \text { under the } \\
\text { multivariate regression model. }\end{array}$} \\
\hline
\end{tabular}

For 427 non-OHCA patients with a critical illness, the TAA remained as an independent determinant (AOR, $1.007 ; P<0.001$ ) of 30-day mortality, after adjustment of four independent predictors (i.e., inadequate source control, fatal comorbidities, bacteremia due to urinary or lower respiratory tract infections) recognized by the multivariate regression model (Table 2 ).

For 2,023 with a less critical illness, each hour delay of the TAA was associated with an average increase in 30 -day mortality of $0.3 \%$ (AOR, $1.003 ; P<0.001)$, after adjustment of independent predictors (nursing home residents, polymicrobial bacteremia, bacteremia due to urinary or lower respiratory tract infections, fatal comorbidities, and comorbidities of liver cirrhosis, malignancy, or chronic obstructive pulmonary diseases).

\section{Discussion}

The present study demonstrated a higher contamination rate of blood cultures and bacteremia incidence in patients with OHCA, compared with those without OHCA at the ED. Notably, significant differences in bacteremia sources, causative microorganisms, and susceptibilities between two patient groups were evident. Compared with the matched non-OHCA patient with community-onset bacteremia, the OHCA patient exhibited a greater likelihood of having bacteremia due to lower respiratory tract infections and a lower likelihood of having urosepsis. Furthermore, more bacteremic events due to anaerobes and streptococci, but fewer events due to E. coli were observed in OHCA patients, and AROs more often existed among such patients. More importantly, the survival benefit of early administration of appropriate antimicrobials in OHCA patients was firstly recognized. Accordingly, the clinical practice of blood culture samplings should be encouraged and the incorporation of broad-spectrum antimicrobials as empirical therapy into the antibiotic stewardship program should be considered as the first aids for OHCA patients. 
Generally, the etiologies of cardiac arrest are presumed to be of cardiac origins, regardless of the presenting cardiac rhythm. However, the fact that non-cardiac origins account for up to $30 \%$ of cardiac arrest population should not be ignored [23]. Recent investigations dealing with non-cardiac etiologies have indicated that asphyxia, respiratory failure, and comorbid respiratory diseases are the leading etiologies [9, 10,24], but information available on infectious etiologies is limited in the literature. In our $\mathrm{OHCA}$ cohort, lower respiratory tract infections were the most common etiology of bacteremia. Moreover, risk factors linked to the development of lower respiratory tract infections, such as the elderly, comorbid neurological diseases, and bed-ridden status, were frequently observed in OHCA patients. Accordingly, we reasonably infer that lower respiratory tract infection is the leading etiology in the frail elderly presenting with OHCA.

Although optimization in the "chain of survival" has been evidenced to improve survival and neurological outcomes in patients with OHCA $[24,25]$, the appropriateness of empirical antimicrobial therapy has not been address during the formulation of strategies to reduce morbidity and mortality. Generally, a delay in the administration of appropriate antimicrobials increases the risk of unfavorable prognoses in adults with community-onset bacteremia, particularly in those who are critically ill [5, 7]. In the latter presenting with cardiac arrest, the prognostic benefit of prompt administration of appropriate antimicrobials was recognized herein. Because of a higher extent of antimicrobial resistance among the etiological pathogens, a longer elapse of the TAA in patients with OHCA who had community-onset bacteremia was not surprised.

Studies have identified several prehospital predictors of survival from OHCA, such as bystander CPR [24, 25], witnessed cardiac arrest [26], EMS transport time [27], ambulance response time [28], and EMS scene time [29]. Although two predictors, namely bystander CPR and witnessed cardiac arrest, were demonstrated to be independent determinants of survival outcomes, the time-related EMS variables, in terms of the response time, transport time, and scene time, were not associated with patient fatality in our cohort. The cohorts in previously established studies differ from those in our study because we included only patients with documented BSIs. Notably, our work is the first to together investigate the effects of pre-hospital factors and antimicrobial therapy on the prognosis of patients experiencing OHCA. We believe that impacts of any time-related EMS variable on patient prognoses might be negligible, because the elapsed TAA was found to be considerably greater than any of the values for time-related EMS variables. Accordingly, efforts to provide prompt treatment for patients with OHCA through the administration of appropriate antimicrobial therapy should be emphasized.

Contaminated blood cultures was widely known to adversely affect health care and medical expenditures, causing unnecessary hospitalizations, antimicrobial administration, and microbiological studies [30]. Furthermore, ED overcrowding is a growing problem worldwide and is associated with blood culture contamination [31], delayed administration of appropriate antibiotics [32, 33], and unfavorable patient outcomes [33]. Patients with cardiac arrest are emergently resuscitated and comprehensively evaluated, which substantially increases the utilization of emergency care resources and the degree of ED 
overcrowding. Therefore, a high contamination rate of blood culture samples in patients experiencing OHCA is not unexpected.

As the study controls, our non-OHCA patients with BSI were selected at a ratio of $6: 1$, according to the arrival timing of each patient with OHCA. Such ED encounters were sufficiently representative of the comparators of community-onset bacteremia for the following reasons. First, the main causative microorganisms, namely E. coli, S. aureus, Streptococcus species, and Klebsiella species, as well as common bacteremia sources, such as urosepsis, intra-abdominal infections, and soft-tissue infections, in the matched non-OHCA patients, were compatible with those in previously published cohorts with community-onset bacteremia $[2,5,7,34]$. Second, the proportion of critically ill patients at bacteremia onset (approximate $20 \%$ ) and the crude mortality rate (approximate 15\%) in the present cohort were also similar to the corresponding data reported in other studies $[2,5,7,34]$.

Of AROs in the community, ESBL-producers have well known adverse effects on the prognoses of bacteremic patients in the literature [35]. Clinical predictors of ESBL-producers in community-onset bacteremia, including healthcare facility residents, urinary catheter use, previous antimicrobial therapy, frequent ED visits [36], and previous hospitalization have been reported [35]. Because of the retrospective nature of the present study, some variables were difficult to assess. However, studies assessing the frequency of ED visits have suggested that it may be correlated with increased healthcare use, serious ill health, and socioeconomic distress $[37,38]$. It was rightfully understandable that etiologic microorganisms in bacteremic patients experencing OHCA were more likely to be antimicrobial-resistant because of frequent utilization of medical care.

Our findings should be interpreted with cautions for several limitations inherent to the study design. First, the recall basis could not be completely abolished because of the retrospective and observational nature. Therefore, two authors recorded information to ensure data accuracy and minimize record discrepancies. Second, the present study was conducted at three hospitals located in southern Taiwan, and our findings may not be applicable to other areas or hospitals. However, we included approximately 3,000 adults that could be regarded as representative of community-onset bacteremia in southern Taiwan. Third, although bacterial translocation raised from intestinal ischemia during the periarrest period might result in secondary bloodstream infections[39], OHCA patients simultaneously experiencing systemic infections were proven for the high level of C-reactive protein herein. Finally, our cohort represents $56 \%$ of all patients experiencing OHCA sampled for blood cultures at EDs. Selection bias may have exerted a certain influence on the results and reflects the inadequate alertness of sepsis as one of the causes of OHCA, as observed in the literature $[9,10]$. We believe a multicenter, prospective study to evaluate the incidence and significance of BSIs are warranted in the future.

\section{Conclusions}

Sampling of blood cultures can be routinely performed for the identification of infectious etiologies of OHCA. Among OHCA patients with community-onset bacteremia, the survival advantage of early 
administration of appropriate antimicrobials and the prevalence of AROs indicates the incorporation of broad-spectrum antimicrobials with anti-anaerobic coverage as the empirical therapy is warranted.

\section{Abbreviations}

ACLS: advanced cardiac life support; ARO: antimicrobial-resistant organism; CLSI: Clinical and Laboratory Standards Institute; CPR: cardiopulmonary resuscitation; ED: emergency department; EKP: Escherichia coli, Klebsiella species, and Proteus mirabilis; EMS: emergency medical service; ESBL: extended-spectrum beta-lactamase; ICU: intensive care unit; IQR: interquartile range; MRSA: methicillin-resistant Staphylococcus aureus; OHCA: out-of-hospital cardiac arrest; TAA: time-to-appropriate antibiotic.

\section{Declarations}

\section{Acknowledgements}

We would like to thank for providing experimental space and facilities by the Diagnostic Microbiology and Antimicrobial Resistance Laboratory, National Cheng Kung University Hospital, College of Medicine, National Cheng Kung University, Tainan, Taiwan. We also thanks Ching-Yu Ho for data collection in SinLau hospital.

\section{Funding}

This study was partially supported by research grants from the Ministry of Science and Technology (MOST 109-2314-B-006-097), the Ministry of Health and Welfare (MOHW109-TDU-B-211-114003), Sin-Lau Hospital (SLH-M106-01, SLH-M107-02, SLH-M108-01, and SLH-109-04), and National Cheng Kung University Hospital (NCKUH-10909031), Taiwan.

\section{Availability of data and materials}

Available from the corresponding author on reasonable request.

\section{Authors' contributions}

CCL and WK conceived the study idea and designed the study. CCL, MH, and CHL supervised the data collection and chart reviews. CCL provided the data of microbiologic analyses. CCL, PC, and CHL provided methodological and statistical advice on study design and data analysis. CCL and WK provided expertise in infectious disease. CCL drafted this manuscript, and WK revised it carefully from a professional point of view. All authors read and approved the final manuscript.

\section{Conflicting interests}

The authors have no conflict of interest to declare.

\section{Consent for publication}


All authors have provided consent for publication of the manuscript.

\section{Ethics approval and consent to participate.}

The study was approved by the institutional review board of National Cheng Kung University Hospital (BER-109-144), Madou Sin-Lau Hospital (SLH 9919-108-006), and Tainan Sin-Lau Hospital (SLH 9919-108009), and the requirement of obtaining informed consent was waived.

\section{References}

1. Bates DW, Pruess KE, Lee TH. How bad are bacteremia and sepsis? Outcomes in a cohort with suspected bacteremia. Arch Intern Med 1995;155:593-8.

2. Laupland KB, Gregson DB, Flemons WW, Hawkins D, Ross T, Church DL. Burden of community-onset bloodstream infection: a population-based assessment. Epidemiol Infect 2007;135:1037-42.

3. Laupland KB, Pasquill K, Dagasso G, Parfitt EC, Steele L, Schonheyder HC. Population-based risk factors for community-onset bloodstream infections. Eur J Clin Microbiol Infect Dis 2020;39:753-8.

4. Valles J, Rello J, Ochagavia A, Garnacho J, Alcala MA. Community-acquired bloodstream infection in critically ill adult patients: impact of shock and inappropriate antibiotic therapy on survival. Chest 2003;123:1615-24.

5. Lee CC, Lee $\mathrm{CH}$, Hong MY, Tang HJ, Ko WC. Timing of appropriate empirical antimicrobial administration and outcome of adults with community-onset bacteremia. Crit Care 2017;21:119.

6. Corona A, Bertolini G, Lipman J, Wilson AP, Singer M. Antibiotic use and impact on outcome from bacteraemic critical illness: the BActeraemia Study in Intensive Care (BASIC). J Antimicrob Chemother 2010;65:1276-85.

7. Lee CC, Lee CH, Yang CY, Hsieh CC, Tang HJ, Ko WC. Beneficial effects of early empirical administration of appropriate antimicrobials on survival and defervescence in adults with community-onset bacteremia. Crit Care 2019;23:363.

8. Berdowski J, Berg RA, Tijssen JG, Koster RW. Global incidences of out-of-hospital cardiac arrest and survival rates: Systematic review of 67 prospective studies. Resuscitation 2010; 81:1479-87.

9. Hess EP, Campbell RL, White RD. Epidemiology, trends, and outcome of out-of-hospital cardiac arrest of non-cardiac origin. Resuscitation 2007;72:200-6.

10. Kitamura T, Kiyohara K, Sakai T, Iwami T, Nishiyama C, Kajino K, et al. Epidemiology and outcome of adult out-of-hospital cardiac arrest of non-cardiac origin in Osaka: a population-based study. BMJ Open 2014; 4:e006462.

11. Kuisma M, Alaspaa A. Out-of-hospital cardiac arrests of non-cardiac origin. Epidemiology and outcome. Eur Heart J 1997;18:1122-8.

12. Coba V, Jaehne AK, Suarez A, Dagher GA, Brown SC, Yang JJ, et al. The incidence and significance of bacteremia in out of hospital cardiac arrest. Resuscitation 2014;85:196-202. 
13. Fang PH, Lin YY, Lu CH, Lee CC, Lin CH. Impacts of Emergency Medical Technician Configurations on Outcomes of Patients with Out-Of-Hospital Cardiac Arrest. Interna J Environ Resear Pub Health 2020;17:1930.

14. Clinical and Laboratory Standards Institute. Performance standards for antimicrobial susceptibility testing; approved standard. Nineteenth informational supplement. CLSI document M100-S19. Wayne, PA: CLSI, 2009.

15. Clinical and Laboratory Standards Institute. Performance standards for antimicrobial susceptibility testing; approved standard. $30^{\text {th }}$ informational supplement. CLSI document M100-S30. Wayne, PA: CLSI, 2020.

16. Panchal AR, Berg KM, Cabañas JG, Kurz MC, Link MS, Del Rios M, et al. 2019 American Heart Association Focused Update on Systems of Care: dispatcher-assisted cardiopulmonary resuscitation and cardiac arrest centers: an update to the American Heart Association Guidelines for Cardiopulmonary Resuscitation and Emergency Cardiovascular Care. Circulation 2019;140:e895e903.

17. Lee CC, Lin WJ, Shih HI, Wu CJ, Chen PL, Lee HC, et al. Clinical significance of potential contaminants in blood cultures among patients in a medical center. J Microbiol Immunol Infect 2007; 40:438-44.

18. Gilbert DN, Moellering RC Jr., Chambers HF, et al. Selected pharmacologic faetures of antimicrobial agents. The Sanford Guide to Antimicrobial Therapy 2020: 78-82.

19. Schellevis FG, van der Velden J, van de Lisdonk E, van Eijk JT, van Weel C. Comorbidity of chronic diseases in general practice. J Clin Epidemiol 1993;46:469-73.

20. McCabe WR. Gram-negative bacteremia. Adv Intern Med 1974;19:135-58.

21. Garner JS, Jarvis WR, Emori TG, Horan TC, Hughes JM. CDC definitions for nosocomial infections, 1988. American journal of infection control 1988;16:128-40.

22. Rhodes A, Evans LE, Alhazzani W, Levy MM, Antonelli M, Ferrer R, et al. Surviving Sepsis Campaign: International Guidelines for Management of Sepsis and Septic Shock: 2016. Intensive Care Med 2017;43:304-77.

23. Lombardi G, Gallagher J, Gennis P. Outcome of out-of-hospital cardiac arrest in New York City. The Pre-Hospital Arrest Survival Evaluation (PHASE) Study. JAMA 1994; 271:678-83.

24. Christensen DM, Rajan S, Kragholm K, Sondergaard KB, Hansen OM, Gerds TA, et al. Bystander cardiopulmonary resuscitation and survival in patients with out-of-hospital cardiac arrest of noncardiac origin. Resuscitation 2019;140:98-105.

25. Iwami T, Nichol G, Hiraide A, Hayashi Y, Nishiuchi T, Kajino K, et al. Continuous improvements in "chain of survival" increased survival after out-of-hospital cardiac arrests: a large-scale populationbased study. Circulation 2009;119:728-34.

26. Hostler D, Thomas EG, Emerson SS, Christenson J, Stiell IG, Rittenberger JC, et al. Increased survival after EMS witnessed cardiac arrest. Observations from the Resuscitation Outcomes Consortium (ROC) Epistry-Cardiac arrest. Resuscitation 2010;81:826-30. 
27. Geri G, Gilgan J, Wu W, Vijendira S, Ziegler C, Drennan IR, et al. Does transport time of out-of-hospital cardiac arrest patients matter? A systematic review and meta-analysis. Resuscitation 2017;115:96101.

28. O'Keeffe C, Nicholl J, Turner J, Goodacre S. Role of ambulance response times in the survival of patients with out-of-hospital cardiac arrest. Emergency medicine journal 2011; 28:703-6.

29. Do Shin S, Kitamura T, Hwang SS, Kajino K, Song KJ, Ro YS, et al. Association between resuscitation time interval at the scene and neurological outcome after out-of-hospital cardiac arrest in two Asian cities. Resuscitation 2014;85:203-210.

30. Hall KK, Lyman JA. Updated review of blood culture contamination. Clin Microbiol Rev 2006;19:788802.

31. Lee CC, Lee NY, Chuang MC, Chen PL, Chang CM, Ko WC. The impact of overcrowding on the bacterial contamination of blood cultures in the ED. Am J Emerg Med 2012; 30:839-45.

32. Peltan ID, Bledsoe JR, Oniki TA, Sorensen J, Jephson AR, Allen TL, et al. Emergency Department Crowding Is Associated With Delayed Antibiotics for Sepsis. Ann Emerg Med 2019;73:345-55.

33. Gaieski DF, Agarwal AK, Mikkelsen ME, Drumheller B, Cham Sante S, Shofer FS, et al. The impact of ED crowding on early interventions and mortality in patients with severe sepsis. Am J Emerg Med 2017;35:953-60.

34. Diekema DJ, Beekmann SE, Chapin KC, Morel KA, Munson E, Doern GV. Epidemiology and outcome of nosocomial and community-onset bloodstream infection. J Clin Microbiol 2003;41:3655-60.

35. Paterson DL, Bonomo RA. Extended-spectrum beta-lactamases: a clinical update. Clin Microbiol Rev 2005;18:657-86.

36. Lee $\mathrm{CH}$, Chu FY, Hsieh CC, Hong MY, Chi CH, Ko WC, Lee CC. A simple scoring algorithm predicting extended-spectrum beta-lactamase producers in adults with community-onset monomicrobial Enterobacteriaceae bacteremia: Matters of frequent emergency department users. Medicine (Baltimore) 2017;96(16):e6648.

37. Hunt KA, Weber EJ, Showstack JA, Colby DC, Callaham ML. Characteristics of frequent users of emergency departments. Ann Emerg Med 2006;48:1-8.

38. Bernstein SL. Frequent emergency department visitors: the end of inappropriateness. Ann Emerg Med 2006;48:18-20.

39. Chalkias A, Scheetz MH, Gulati A, Xanthos T. Periarrest intestinal bacterial translocation and resuscitation outcome. J Crit Care 2016, 31:217-20.

\section{Figures}


Total 1,021,177 adult encounters in the EDs of three hospitals between January 2008 and December 2017

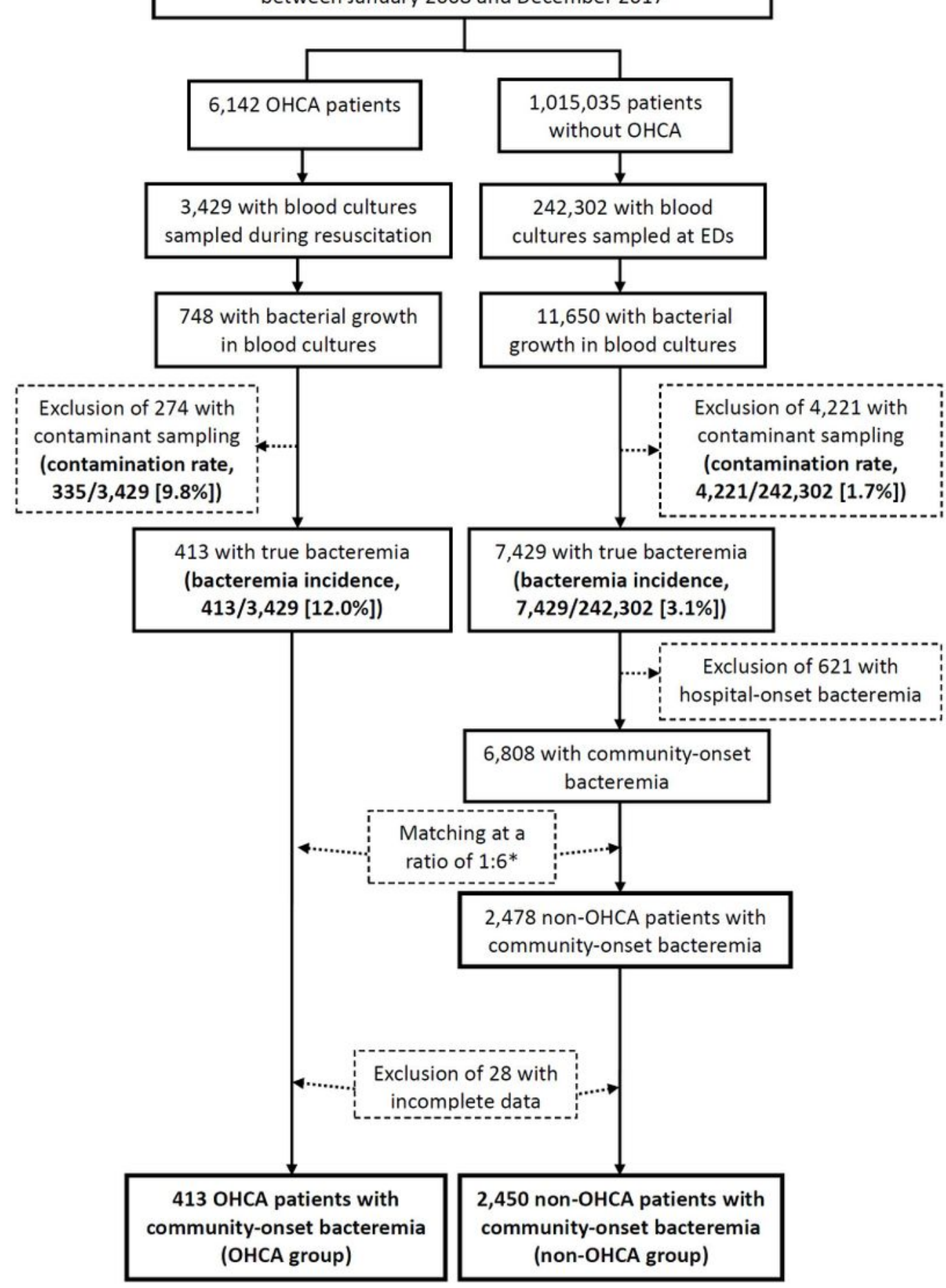

\section{Figure 1}

The flowchart of patient selections. $\mathrm{ED}=$ emergency department; $\mathrm{OHCA}=$ out-of-hospital cardiac arrest. *Six non-OHCA patients temporally near the ED arrival of OHCA visits were matched. 


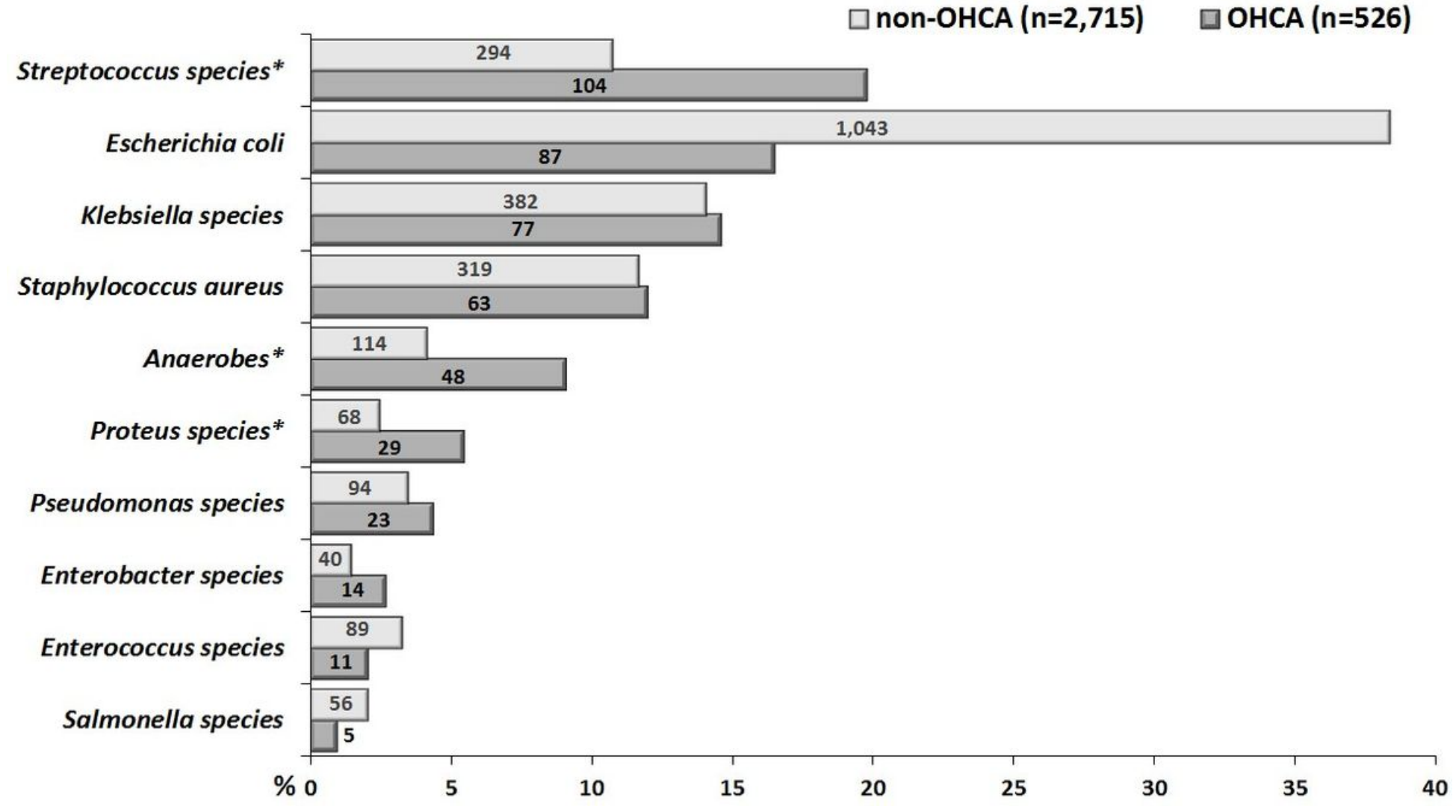

Figure 2

The distribution of causative microorganisms of community-onset bacteremia in adults with and without out-of-hospital cardiac arrest (OHCA and non-OHCA)\#. * Indicated the significant difference (i.e., $\mathrm{P}<0.05)$ between two groups. \# Numbers in the bar indicated the isolate number. 


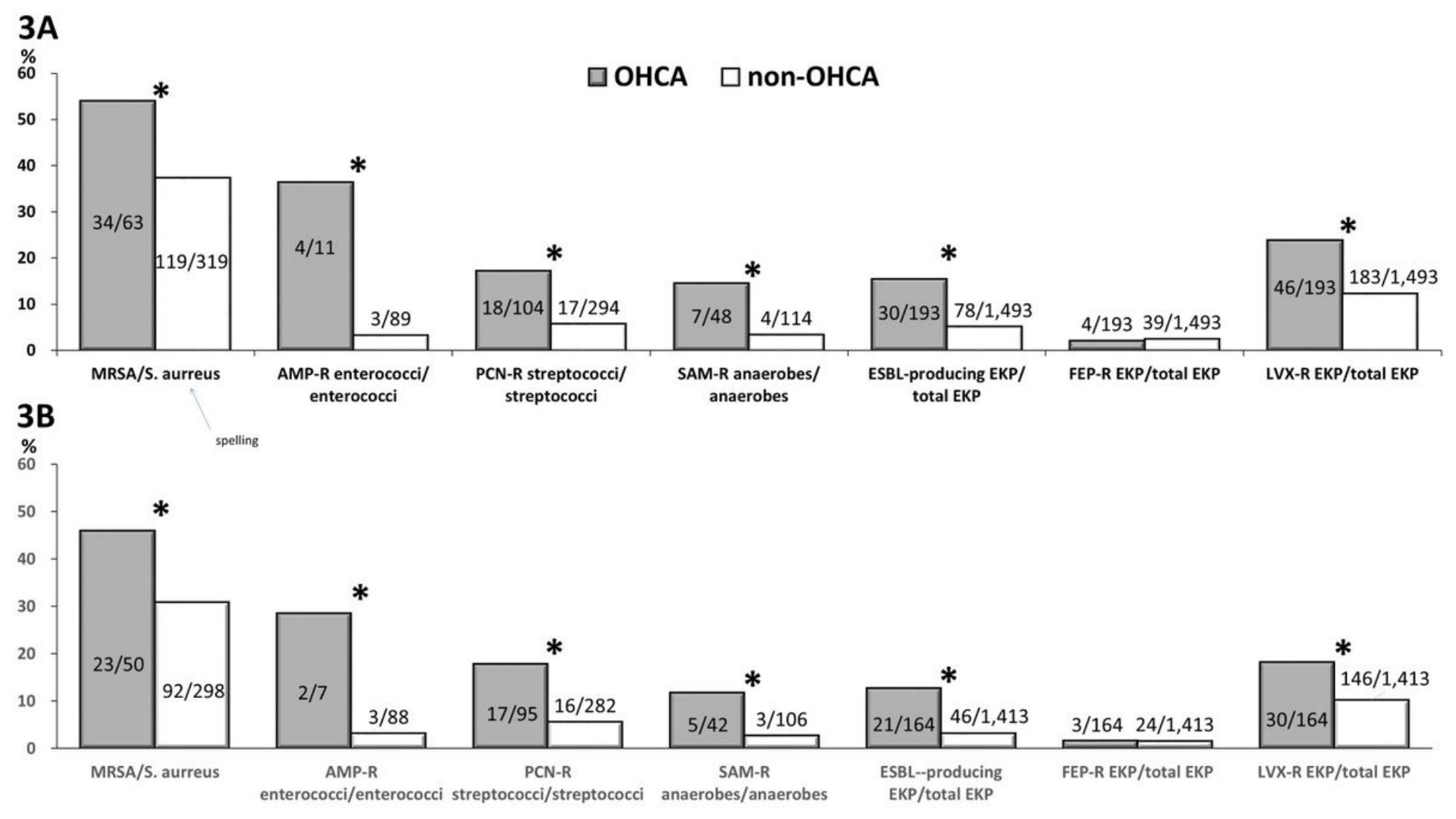

Figure 3

The comparison of the susceptibility of community-onset bacteremia-causing microorganisms between the OHCA and non-OHCA patients in the overall cohort (Figure 3A) and home-living patients (Figure 3B) \#. AMP = ampicillin; EKP = Escherichia coli, Klebsiella species, and Proteus mirabilis; ESBL = extendedspectrum beta-lactamase; $L V X$ = levofloxacin; MRSA = methicillin-resistant Staphylococcus aureus; $P C N=$ penicillin; $\mathrm{R}$ = resistant; $\mathrm{SAM}=$ ampicillin/sulbactam. * Indicated the significant difference (i.e., $\mathrm{P}<0.05$ ) between two groups. \# The denominators and numerators respectively indicate the isolate numbers of all microorganisms and antimicrobial-resistant pathogens. 


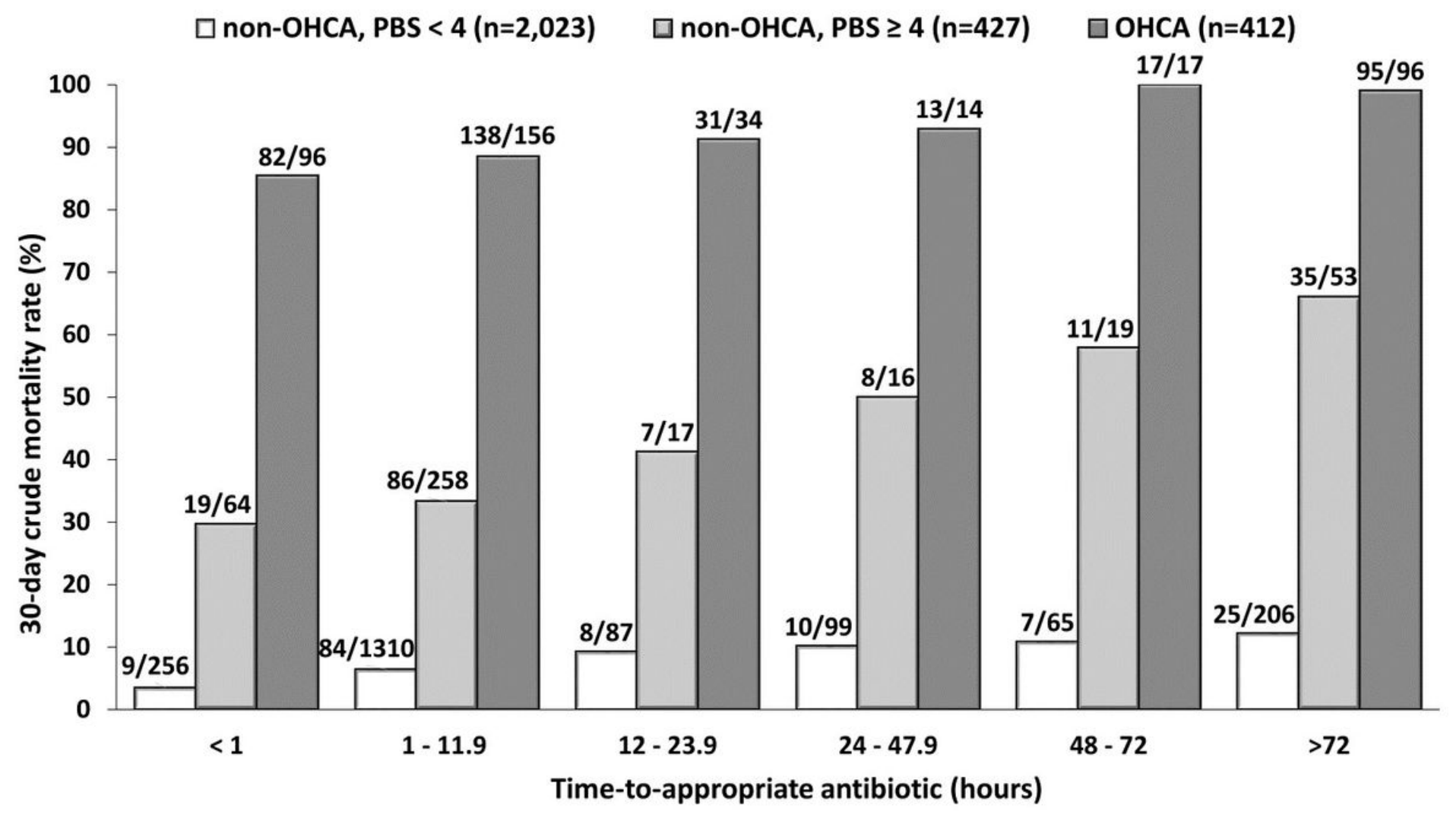

Figure 4

A positive trend of the time-to-appropriate antibiotic in 30-day crude mortality rate, categorized into the patients experiencing out-of-hospital cardiac arrest (OHCA), non-HOCA patients with a critical illness (a Pitt bacteremia score $\geq 4$ ), and those with a less critical illness (a Pitt bacteremia score $<4$ )*. *The denominators and numerators respectively indicate case numbers of crude 30 -day mortality and all patients in varied categories of the time-to-appropriate antibiotic. 